\title{
Tethered insect flight: A system for automated data processing of behavioral events
}

\author{
D. L. CHAMBERS, J. L. SHARP, and T. R. ASHLEY \\ Insect Attractants, Behavior and Basic Biology Research Laboratory, Agricultural Research Service, \\ United States Department of Agriculture, Gainesville, Florida $\$ 2604$
}

Flight mills to which flying insects are tethered, and which allow measurement of locomotion in a circular path, are used to measure flight capability in terms of frequency, duration, velocity, and distance. Also, they are adaptable to measurement of physiological processes such as respiration and energy consumption. We describe a flight-mill system with floating magnets and Teflon ${ }^{\oplus}$ bearings in its rotor that minimize frictional drag. Rotation (of the mill rotor) is transduced by interruption of a light beam to a phototransistor, which produces a signal monitored by a specially constructed event recorder. The event recorder temporarily stores the data from each mill and then transmits them in proper format for permanent storage on seven-track magnetic tape. The system allows simultaneous data collection from 18 individual insects and provides a computerized interpretation of their performance. The result is an analysis of all the significant parameters of flight that can be derived by measuring duration, velocity, distance, and frequency.

Devices to which insects are tethered for study of flight characteristics have a fairly long history characterized by an increasing degree of innovation in the apparatus and in the complexity of the data that can be derived. Flight mills (turnabouts or roundabouts), in particular, have often been used in examining flight ability, usually in terms of flight frequency, duration, speed, and range (Henneberry, Kishaba, Igbal, \& Klinger, 1968; Koerwitz \& Pruess, 1964; Krogh \& Weis-Fogh, 1952; Sharp, Leppla, Bennett, Turner, \& Hamilton, 1974). Also, physiological parameters have been studied with flight mills (Bennett \& Bordon, 1971; Hocking, 1953; Michel, 1973; Yurkiewicz, 1967; Yurkiewicz \& Smyth, 1966).

Flight mill designs are based on a common concept: Test insects are fastened to the end of a horizontal arm that rotates on a vertical axle in response to insect flight. The rotation of the arm around the axle is used to analyze flight behavior in terms of frequency, duration, velocity, and distance.

We required a system that provided fully automated data processing of flight performance. Such a system is described here. It includes 18 flight mills, a controller to sample the activity of each mill, and a computercompatible magnetic tape unit to record the data. The recorded data are computer processed (Ashley, Sharp, \& Chambers, 1976) to obtain a variety of statistics related to the activity of each mill and to subgroups of mills.

\section{METHOD}

\section{Principle of Operation}

Functionally the system is an 18-channel event recording device (see Figure 1). Each channel consists of a flight mill, a transducer to indicate when each mill has made one revolution, and a storage element to hold that count a short time, until it is recorded on magnetic tape. Operation of the system is controlled by a controller and a power supply.

The flight mill (Figure 2) consists of an adaptation of the system reported by Chambers and O'Connell (1969) in which magnetic floatation was used to provide nearly friction-free rotation. Two disk-shaped magnets $(F)$ are suspended on a rigid shaft (A), with like magnetic poles facing each other. Magnetic repulsion allows the upper magnet to "float," eliminating verti-

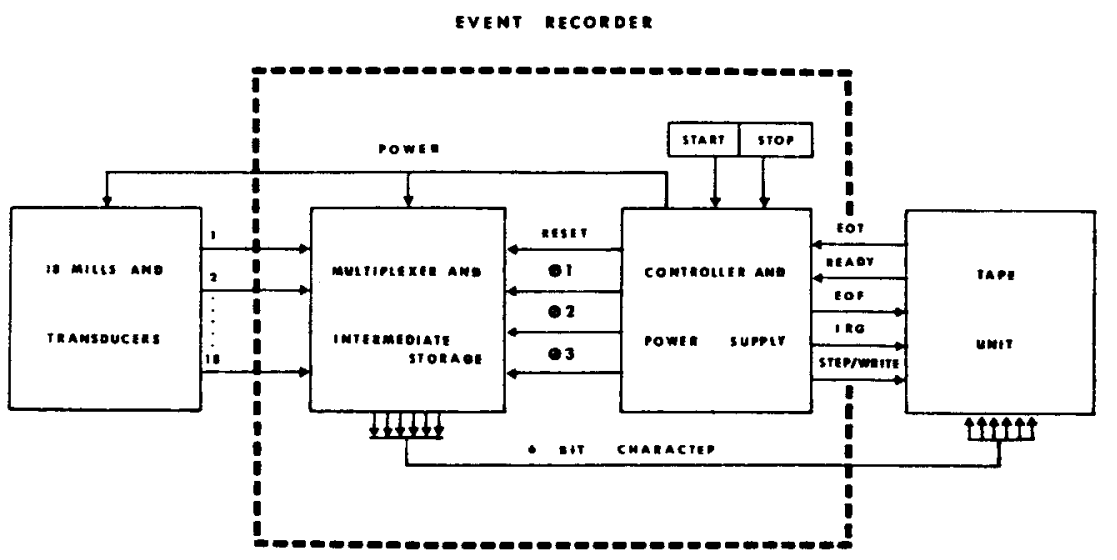

Figure 1. Block diagram indicating flight mill input to the event recorder, which contains a multiplexer and intermediate storage wave form controlled by a controller and power supply that transduces gated stored signals to a computer-compatible magnetic tape unit. 


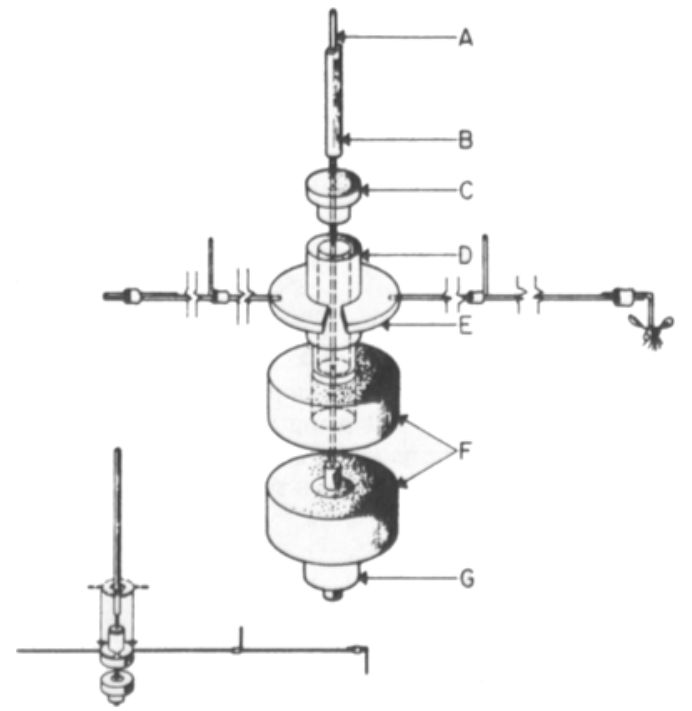

Figure 2. Flight mill, showing: A-hypodermic syringe needle serving as the axle; $B$-brass tubing providing rigidity and support; $\mathrm{C}$-Teflon hub cap drilled for minimal friction against the axle; D-Teflon hub counter-drilled for no axle contact except minimally at lower end; E-rotor, consisting of notched washer and syringe needle arms with phototransducer interrupters and insect mounts; F-magnets with facing like poles, allowing the upper to float freely about the axle;G-Teflon hub and brass tube which affix lower magnet to end of axle. Inset shows mounting of rotor to hub.

cal friction points; the only points of contact are lateral against the vertical shaft. The magnets are center-drilled to accept Teflon ${ }^{\circledR}$ hubs (D, G). The lower tightly fits the shaft; the upper (free to rotate about the shaft) is counter-drilled and fitted with a cap (C) so that only the terminal ends of the hub contact the shaft, which further reduces friction. Also, the upper Teflon hub is longer $(2 \mathrm{~cm})$, to reduce rocking on the shaft, and the holes in its ends are about $.002 \mathrm{~cm}$ larger than the shaft in order to minimize contact without excessive play.

The shaft (A) is a stainless steel hypodermic syringe tube $(.0813 \mathrm{~cm})$ selected for the rigidity it provides while retaining small size and, hence, minimal friction at the hub. Rigidity of the system is further increased by inserting the shaft from a point just above the hub through a brass tube of greater diameter (B) that accepts it tightly, reduces sway, and provides an upper point of attachment via clamps. A similar flight mill rotor was described by Schoenleber et al. (Note 1). At the time of their report, we were developing a device based on the recommendation made by a watchmaker familiar with the use of such bearings in large clocks (where the system is called a taut-wire magnetic bearing). Von Remund and Boller (1975) developed a similar rotor system.
The rotor arm (E) is constructed of 25-ga stainless steel hypodermic tubing rigid enough to preclude droop and bounce and thin enough and lightweight enough to minimize aerodynamic and inertial drag. The arm to which the insect is attached and a counterbalanced arm are fastened to a steel washer by pressing the arms into holes drilled in the rim of the washer. The washer is notched to allow insertion around the shaft so it can be lowered onto the upper magnet, where it is held magnetically (Figure 2). Insects are mounted on the rotor arm by using rubber sleeves (Chambers \& O'Connell, 1969).

The positioning of test insects on the rotor must be considered and standardized. Earlier empirical data indicated that optimal performance was obtained by positioning the insect with the body tipped, head down, at an angle of 12-14 deg from horizontal. Subsequently, acoustical and physical analyses (J. C. Webb of this laboratory, unpublished data) showed that the greatest thrust of power generated by flight movements occurred 13 deg below and behind a horizontally positioned Caribbean fruit fly, Anastrepha suspensa (Loew). Thus, we continue to use the head-down posture in mounting insects, and it has proven to be most satisfactory for the Diptera and Lepidoptera tested.

Magnet size, hub size, and rotor length can be varied to provide inertial and aerodynamic drag coefficients and angular flight velocities most suitable for the size and flight capabilities of the insects being studied. We have constructed two hub sizes and three rotor sizes. The magnets for each of the two hubs measure $1.2 \mathrm{~cm}$ o.d. (mean total hub weight $=2.1 \mathrm{~g}$ ) and $2.0 \mathrm{~cm}$ o.d. (mean weight $=9.2 \mathrm{~g}$ ), respectively. The three rotor arm lengths (centimeters) and weights (grams) are, respectively, 20.0 and $1.5,21.5$ and 3.0 , and 31.85 and 5.1. The longest rotor circumscribes a circle $1 \mathrm{~m}$ in diam.

Rotation of the flight mill rotor is transduced by interruption of a light beam from a No. 222 miniature lamp to a FPT-100 phototransistor. This interruption occurs because two small vertical rods are attached to the two opposing rotor arms, equidistant from the hub (Figure 2). As the insect makes one revolution of flight, the light path is interrupted twice. Two pulses from the transducer are required to turn the intermediate storage element on.

The intermediate storage element (Figure 3) has two states: 1 and 0 . When it is in the 1 state, it is said to be on and indicates that one revolution of the mill has occurred.

The 18 storage flip-flops are multiplexed (Figure 4) in three groups of six. Each group of six elements is recorded as one character on the magnetic tape. Immediately after each character is recorded, the associated mill storage elements of those mills that have completed one revolution during the preceding sample period are automatically reset to 0 . At the end of each $1 / 2-\mathrm{sec}$ interval, three 6-bit characters are transmitted by the controller (Figures 1 and 4 ) to the tape unit. The controller section is hard wired to record 120 samples $(1 \mathrm{~min})$ per record. An IRG (interrecord gap) command is sent after the last character of the 120 th sample. An EOF (end-of-file) command is generated upon the completion of the record in progress after the stop button is

INTERMEDIATE STORAGE ELEMENT

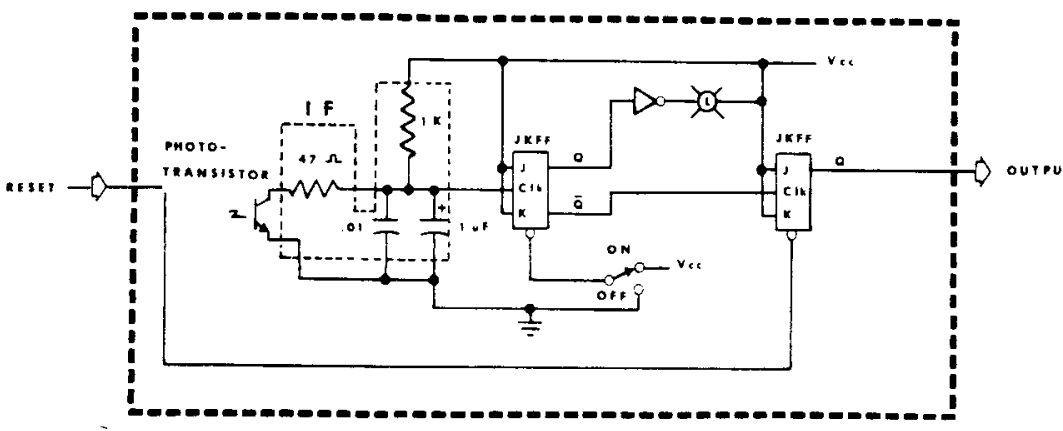

Figure 3. Block diagram of a single representative intermediate storage element indicating the input filter which eliminates spurious signals and a MS flip-flop providing output to the multiplexer. 


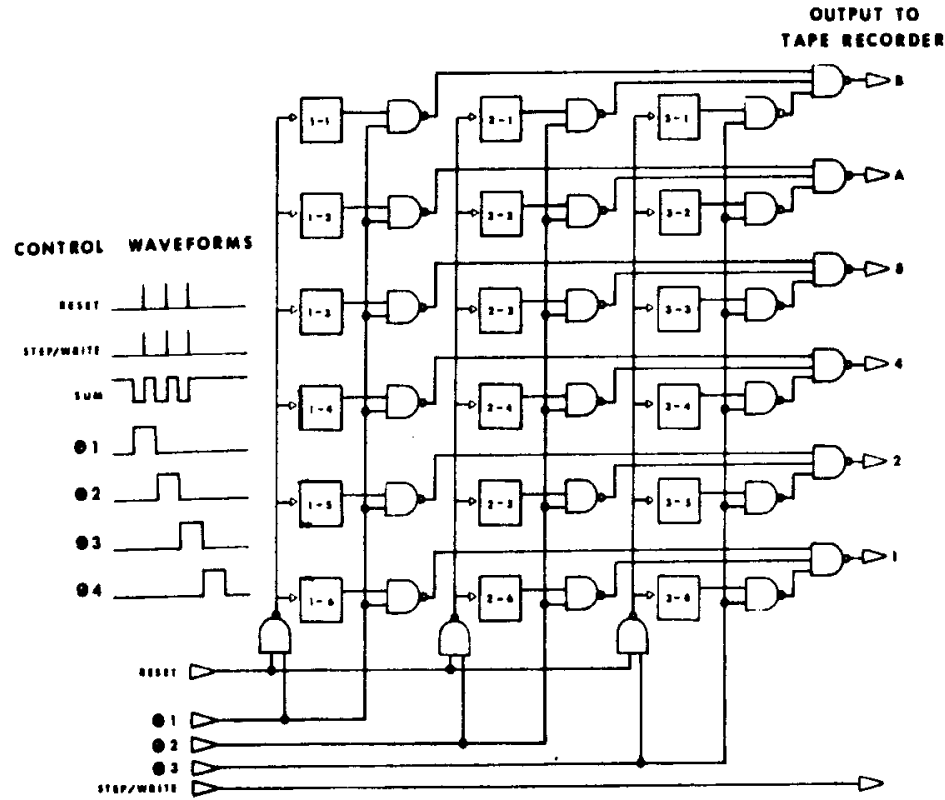

Figure 4. Block diagram of the multiplexer, 6-bit characters being gated to the tape unit and being signaled by the control wave forms $01,02,03$, and 04 , plus reset, step/write, and sum signals.

pushed. The encounter of an end-of-tape marker on the magnetic tape will have the same effect as pushing the stop button on the controller.

The tape unit is an incremental (records character by character, as opposed to record by record) seven-track magnetic tape recording device capable of producing computer-compatible output. Since the recorder can only indicate that an event-one revolution of the mill-has occurred, the sampling period must be short enough to preclude the omission of multiple events. The sampling period has therefore been set at two samples/sec, and 120 samples, equivalent to $1 \mathrm{~min}$ of running time, are recorded as a single record on the magnetic tape. One standard $19-\mathrm{cm}$ reel of magnetic tape can record up to $100 \mathrm{~h}$ of continuous activity.

A load command resets the data register, advances the tape to the beginning-of-tape marker, and inserts a beginning-of-tape gap of about $7.62 \mathrm{~cm}$. A ready command allows the tape unit to accept data if the tape is loaded. The EOF push-button command causes the tape unit to sequentially write an RRC (longitudinal redundancy check character), a $9.525-\mathrm{cm}$ file gap, a file mark, and a $1.905-\mathrm{cm}$ interrecord gap. The rewind command causes the tape to rewind to the beginning-of-tape marker. Pushing the rewind button a second time rewinds the tape onto the supply reel.

\section{Circuit Description}

Input circuit. The phototransistor (Figure 3) must be capable of sinking at least $5 \mathrm{~mA}$. The controller contains 1-kohm pull-up resistors to supply power to the photodetectors. The function of the input filter (Figure 3, IF) is to remove spurious signals. This is required to maintain an accurate count of mill rotation by preventing electrical noise disturbance of the timing and control logic. The input is first RC filtered and then applied to the clock input of an MS (master-slave) flip-flop. The 18 channel switches (one for each input) on the front panel hold the input filter flip-flops in the reset state when they are in the off position.

Intermediate storage and multiplexer. The intermediate storage (Figure 3 ) consists of $18 \mathrm{MS}$ flip-flops arranged in a 3 by 6 array corresponding to Inputs $1-6,7-12$, and 13-18. The multiplexer (Figure 4) presents at its output a 6-bit character representing one-third of the array. Series 74 TTL (transistor- transistor logic) NAND gates are used as line drivers to the tape unit.

Controls. Timing is derived from the $60-\mathrm{Hz}$ ac power line. A positive full rectified sine wave obtained from the output of the main power transformer (Figure 4) is RC filtered and passed through an MS flip-flop to produce a $60-\mathrm{Hz}$ square wave. The $60-\mathrm{Hz}$ signal is frequency divided to provide a maximum timing duration of $1 \mathrm{~min}$. The frequency dividers produce an unweighted but symmetrical output that is quite convenient for clock pulse generation. Spurious modes are suppressed by feedback. The clock flip-flops are forced to the zero state until the run condition is enabled.

The signals $\emptyset 1, \emptyset 2, \emptyset 3$, each $16 \mathrm{msec}$ long (from the frequency divider network), are used sequentially to access each of the three 6-bit intermediate storage locations and present the contents to the tape-unit data lines. The address lines also gate a common reset line to the same element that is being presented at the output. The signal sum is used to generate a 10 -microsec step/write command to the tape unit $8 \mathrm{msec}$ after the data lines are presented. The $\emptyset 3$ signals also gate the $100-\mathrm{msec}$ reset pulse to the addressed 6-bit storage immediately after its contents have been entered into the tape unit. The signal $\varphi 4$, which occurs immediately after 03 , is gated out every second to produce a $10-\mathrm{msec}$ IRG command to the tape unit.

The tape unit can write incrementally one 6-bit character (plus one parity bit generated within the tape unit) every $10 \mathrm{msec}$. The IRG requires $175 \mathrm{msec}$. Therefore, the controller was constructed to require characters to be written no closer than $17 \mathrm{msec}$ apart and allows about $450 \mathrm{msec}$ for an IRG.

When power is applied to the controller, it always comes on in the run mode, that is, it starts accepting data. This was done so that intermittent power failures would leave the system running. At most, one or two records (minutes of data) would be lost, rather than all subsequent data.

The run flip-flop is turned on by the start push-button of the controller. (The tape unit must first be readied to make the start switch operative.) The stop push-button turns on the last record flip-flop. The on output from the flip-flop causes the gated $\emptyset 4$ to generate an EOF command to the tape unit rather than the interrecord gap command that is usually generated. The IRG pulse is also used to turn the run flip-flop off. In this way, the last record is completed before data taking is stopped 
Push-buttons on the tape unit are disabled during the run time.

Power supply. The $110-\mathrm{V} \pm 10 \%$ ac $60-\mathrm{Hz}$ power line is switched at the front panel. A pilot light is powered from the $5-\mathrm{V}$ dc (Vcc) supply. There is also an auxiliary power supply in the controller chassis.

The $5-\mathrm{V}$ dc $(\mathrm{Vcc})$ power is obtained by supplying unregulated $10-\mathrm{V}$ dc power to a series regulator. The regulator is mounted on a printed circuit card with the final power series pass transistor heat sinked to the horizontal chassis panel. The $5-\mathrm{V}$ regulator has a $\pm 10 \%$ adjustment potentiometer on the card and is protected from short circuit and overload by an automatic current fold-back feature. The auxiliary $2.5-\mathrm{V}$ unregulated supply is provided for powering the transducer lamps.

\section{EXPERIMENTAL DATA}

Results are reported of a simple test designed to evaluate the effect of varying hub sizes and rotor lengths on the performance of male Anastrepha suspensa. Geometries of the hubs allowed us to compare large hubs with long or medium rotors and small hubs with medium or short rotors. Flies 11-14 days old were flown under controlled conditions of $26^{\circ} \mathrm{C}$ and $65 \%$ relative humidity for $3 \mathrm{~h}$ in a series of experiments in which the four combinations of rotor and hub size were compared. Performance was measured by percentage of time fly. ing, total distance flown, overall flight velocity, and average velocity of the fastest flight (a flight was arbitrarily defined as activity not interrupted by a rest period longer than $2.5 \mathrm{sec}$ ).

The data for the first three performance categories are presented in Table 1. Statistical analysis showed no differences when hubs and rotors were interchanged. However, a higher average velocity of the fastest flight was attained by males attached to the larger hubs and the longest and medium arms, though this statistic has limited significance because it represents only the single fastest flights in each test. Perhaps the results indicate that this insect is more suited for larger flight radii, with lower angular velocities, though the conditions are not highly critical. As Hocking (1953) points out, most energy loss on a mill system is metabolic rather than aerodynamic. Thus, decisions on hub and rotor size may be governed more by the logistics of mounting, inertial weight, and flight velocities amenable to system reaction time than by considerations of aerodynamics and angular velocities.

The lack of variation in the three most critical performance categories for the different hub and rotor combinations indicates the low coefficient of friction provided by magnetic flotation and confirms the adequacy of the mill design.

\section{DISCUSSION}

A variety of harnesses and adhesives have been used with flight mill systems for affixing the insect to the rotor. The selections seem to be based on individual choice and suitability. A principal variation among described mills is in the bearing system for the vertical
Table 1

Flight Ability (Mean \pm SE) of Anostrepha suspensa Males Flown for $3 \mathrm{~h}$ on a Mill System Equipped with Different Magnets and Rotors

Flight Performance Categories

\begin{tabular}{|c|c|c|c|}
\hline \multirow[b]{2}{*}{ Treatments } & \multicolumn{3}{|c|}{ Flight Performance Categories } \\
\hline & $\begin{array}{l}\text { \% Time } \\
\text { Flying }\end{array}$ & Distance (m) & $\begin{array}{l}\text { Overall } \\
\text { Flight } \\
\text { Velocity } \\
\text { (m/min) }\end{array}$ \\
\hline $\begin{array}{l}\text { Large magnet }+ \\
31.85-\mathrm{cm} \text { rotor }\end{array}$ & $49 \pm 5$ & $3400 \pm 335$ & $38 \pm 1$ \\
\hline $\begin{array}{l}\text { Large magnet + } \\
21.5-\mathrm{cm} \text { rotor }\end{array}$ & $42 \pm 3$ & $3290 \pm 277$ & $40 \pm 1$ \\
\hline $\begin{array}{l}\text { Small magnet }+ \\
21.5-\mathrm{cm} \text { rotor } \\
\text { Small magnet }+\end{array}$ & $48 \pm 7$ & $3625 \pm 588$ & $39 \pm 4$ \\
\hline $20.0-\mathrm{cm}$ rotor & $38 \pm 4$ & $2772 \pm 367$ & $39 \pm 2$ \\
\hline
\end{tabular}

Note-There were no significant differences at the 5\% probability level among means in the same column by Duncan's new multiple range test.

axle. Ball (Krogh \& Weis-Fogh, 1952), glass (Smith \& Furniss, 1966), jeweled (Rowley, Graham, \& Williams, 1968; Schoenleber et al., Note 1), and Teflon terminal bearings (Kishaba, Henneberry, Hancock, \& Toba, 1967) have been used with lower, upper, or dual points or with flotation of the rotor in or on a magnetic field (Chambers \& O'Connell, 1969; Cullis \& Hargrove, 1972; Schoenleber et al., Note 1).

A more critical variation is evident in the methodologies used for data accumulation and processing. Rotation of the arm through a known circumference during a given period provides data on distance flown and velocity. Counts of the numbers of rotations have been made manually on individual mills (Chambers \& O'Connell, 1969), observationally on an array of mills (Yates, 1969), automatically on kymographic (Hocking, 1953) or strip chart recorders (Borden \& Bennett, 1969), and via digital counters tripped by interruption of photocellular transducers (Kishaba et al., 1967; Smith \& Furniss, 1966), alone or in combinations (Rowley et al., 1968).

Our system is essentially an event recorder and, as such, is potentially amenable to many behavioral studies in which automated data processing of events is desirable. Also, the components of the system are separable for adaptation to types of tests other than those described. For example, the recorder can be modified to accept other forms of events and other transduction systems. Similarly, the flight mill can be used independently, interfaced with other data accumulation schemes, or used in studies of an entirely different nature such as optometric, respirometric, or acoustic analysis. In this laboratory, individual mills have been used in conjunction with sound recording and analysis equipment for basic studies of the physics of flight sound (Webb, Sharp, Chambers, \& Benner, 1976).

The flight mill system we describe is suitable for behavior studies of virtually any flying insect and 
adaptable for use under most controlled environmental conditions. The most salient features are low friction, flexibility, and high data output, with low cost in terms of man hours in data transduction. The system allows simultaneous comparison of a relatively large number of insects (18) compared with other systems and, more importantly, interfaces the experimental units directly with an event recorder that transduces results directly to computer-compatible analytical procedures. For studies with large numbers of insects and large numbers of tests, the cost of the unit more than offsets the man-hour costs of systems that do not provide automatic data processing.

Answers to questions, a list of components and suppliers, as well as blueprints of the event recorder (at cost of duplication) can be obtained from the senior author. We cannot confirm the cost of the components to scientists located elsewhere, but the following figures can act as guidelines for those who might wish to consider constructing such a system. (1) Flight mill and transducer components, less labor costs $=\$ 6$ $\$ 8 /$ mill. (2) Event recorder including custom design and construction $=\$ 2,500$ (parts and materials approximately $\$ 100$ ). (3) Tape recorder $=\$ 1,800$. (4) Computer time = approximately $\$ 1$ for analysis of a 1 -h test.

\section{REFERENCE NOTE}

1. Schoenleber, L. G.. White, L. D., \& Butt, B. A. Flight mill system for studying insect behavior. United States Department of Agriculture, Agricultural Research Service Technical Bulletin, 1970, 42-164, 1-7.

\section{REFERENCES}

Ashley, T. R.. Sharp, J. L. \& Chambers, D. L. Tethered insect flight: A computer program to analyze behavioral events. Behavior Research Methods \& Instrumentation. 1976. 8. $385-387$.

Bennett, R. B., \& Bordon, J. H. Flight arrestment of tethered Dendroctonus pseudotsugae and Trypodendron lineatum (Coleoptera: Scolytidae) in response to olfactory stimuli. Annals of the Entomological Society of America, 1971, 64, 1273-1286.

Borden, J. H., \& Bennett, R. B. A continuously recording flight mill for investigating the effect of volatile substances on the flight of tethered insects. Journal of Economic Entomology, 1969, 63, 782-785.

Chambers, D. L., \& O'Connell, T. B. A flight mill for studies with the Mexican fruit fly. Annals of the Entomological Society of America. 1969, 62. 917-920.

Cullis, N. A., \& Hargrove, J. W. An automatic device for the study of tethered flight in insects. Bulletin of Entomological Research, 1972, 61, 533-537.

HenneberRy, T. J., Kishaba, A. N., Igbal, M. Z. \& KLINGER, B. B. Reproduction. longevity, and flight of cabbage looper moths treated topically with tepa. Journal of Economic Entomology. 1968, 61, 1536-1540.

Hocking, B. The intrinsic range and speed of insect flight. Transactions of the Royal Entomological Society of London. 1953, 10. 223-345.

Kishaba, A. N., Henneberry, T. J., Hancock, P., \& TOBA, H. H. Laboratory technique for studying flight of cabbage looper moths and the effects of age. sex, food. and tepa on flight characteristics. Journal of Economic Entomology. 1967. 60, 359-366.

Koerwitz, F. L., \& Pruess, K. P. Migratory potential of the army cutworm. Joumal of the Kansas Entomological Society, 1964, 37, 234-239.

KROGH. A., \& WEIS-Fogh. T. A roundabout for studying sustained flight of locusts. Jourmal of Experimental Biology, 1952. 29. 211-219.

Miches, R. Influence de la lumiere et de l'obscurite sur l'induction au vol et sa cessation chez le criquet pelerin Schistocera gregaria (Forskal) eleve en isolement ou en groupes denses. Behaviour, 1973, 45, 1-11.

Rowley, W. A., Graham, C. L.. \& Williams, R. E. A flight mill system for the laboratory study of insect flight. Annals of the Entomological Society of America, 1968 , $61,1507.1514$

Sharp, J. L., Leppla, N. C., Bennett, D. R., Turner, W. K., \& Hamilton, E. W. Flight ability of Plecia nearctica in the laboratory. Annals of the Entomological Society of America, 1974, 67, 735-738.

Smith, H. W., \& FuRniss, M. M. An automatically recording insect flight mill. Canadian Entomologist. 1966, 98, 249-252.

Von Remund, U., \& Boller, E. F. Qualitatskontrolle bei Insekten: Messung von Flugparametern. Zeitschrift für angewandte Entomologie. 1975. 78, 113-126.

Webb, J. C.. Sharp, J. L., Chambers, D. L., \& Benner, J. C. Acoustical properties of the flight activities of the Caribbean fruit fly. Journal of Experimental Biology, 1976, in press.

YATES, R. W. Flight ability and flight metabolism with relation to age in the fruit thy Dacus tryoni. A thesis submitted as part of the requirement for the Degree of Bachelor of Science with honors within the University of Sydney, Sydney, Australia, 1969.

YURKIEwICz, W. J. A respirometer tlask for measuring oxygen consumption during flight on a turnabout. Annals of the Entomological Society of America, 1967, 60, 1122-1123.

YURKIE wicz, W. J., \& SMYTh. T.. JR. Effect of temperature on flight speed of the sheep blowfly. Journal of Insect Physiology, 1966, 12, 189.194.

(Revision received February 13, 1976.) 